\title{
The casein peptide Asn-Pro-Trp-Asp-GIn enforces the intestinal tight junction partly by increasing occludin expression in Caco-2 cells
}

\author{
Hatsumi Yasumatsu and Soichi Tanabe* \\ Graduate School of Biosphere Science, Hiroshima University, Higashi-Hiroshima 739-8528, Japan
}

(Received 10 December 2009 - Revised 15 February 2010 - Accepted 25 March 2010 - First published online 19 May 2010)

We have demonstrated previously that Asn-Pro-Trp-Asp-Gln (NPWDQ, amino acids $107-111$ of $\alpha s_{2}$-casein) inhibited allergen permeation, such as that demonstrated by ovalbumin, using Caco-2 cells as an in vitro human intestinal epithelial model and in mouse jejunal and ileal loops ex vivo. In the present study, the mechanism underlying this inhibitory activity was examined in Caco- 2 cells. Transepithelial resistance value increased in response to the addition of increasing NPWDQ concentrations $\left(10^{-6}-10^{-4} \mathrm{M}\right)$, which suggests that this peptide enhanced epithelial barrier function. Next, changes in mRNA expression by the addition of NPWDQ $\left(10^{-6} \mathrm{M}\right)$ were analysed in Caco-2 cells using the microarray method. NPWDQ up-regulated the expression of the occludin gene in cells, but the level of the genes of the claudin family and zonula occludens-1 (ZO-1) was unchanged. Increased protein expression of occludin, but not of claudin-1 or of ZO-1, was also observed. Therefore, it is suggested that NPWDQ up-regulated the expression of occludin in particular and enforced the tight junction barrier. These data imply that a food-derived peptide can fine-tune the epithelial barrier.

Casein peptides: Tight junctions: Occludin: Caco-2: Microarrays

Allergic diseases are the most common of all immunologically mediated conditions, and are increasing in prevalence in most developed countries. Food allergy has been thought to involve an excessive immune reaction against allergens permeating from the intestinal tract. Theoretically, the intestinal epithelium acts as a barrier restricting the permeation of macromolecules. However, a small proportion $\left(10^{-3}-10^{-4}\right)$ of dietary proteins can cross the epithelium to access and activate effector cells, resulting in food allergy ${ }^{(1)}$.

It is hypothesised that altered intestinal barrier function permits increased transport of dietary antigens across the intestinal barrier and exposure of dietary antigens to the mucosal immune system, leading to the development of a dietary antigen-specific response ${ }^{(2)}$. Consistent with this hypothesis, intestinal permeability in infants with food allergy, as assessed by the lactulose:mannitol ratio in urine, was significantly increased compared with that seen in healthy young children $^{(3)}$. It is therefore conceivable that enhancing the barrier function of the intestinal epithelium would help prevent food allergy.

Our research team has established an in vitro system to evaluate allergen permeation using differentiated Caco-2 cells grown on a permeable filter ${ }^{(4)}$. Caco- 2 cells, human adenocarcinoma cell line derived from a colon carcinoma, have been used as an in vitro model for various purposes. For example, we estimated the transepithelial transport of dietary components, such as the flavonoid hesperidin, in the
Caco-2 cell monolayer ${ }^{(5)}$. Caco-2 cells differentiate under standard culture conditions to form confluent monolayers, and acquire many features of absorptive intestinal cells during culture. These cells spontaneously exhibit various enterocytic characteristics, including the formation of intercellular tight junctions (TJ).

Using this TJ model, we have reported that enzymemodified cheese (EMC) inhibited the permeation of the representative food allergen ovalbumin (OVA). Asn-Pro-TrpAsp-Gln (NPWDQ, amino acids 107-111 of $\alpha s_{2}$-casein) was isolated from the hydrolysate and identified as one of the responsible peptides for this inhibitory activity ${ }^{(4)}$. Intestinal permeability of both fluorescein isothiocyanate-conjugated dextran and horseradish peroxidase decreased in Caco-2 cells by the addition of NPWDQ ${ }^{(6)}$. We also examined if NPWDQ inhibited allergen permeation ex vivo using rats with intestinal inflammation induced by subcutaneous injections of indomethacin. When OVA was injected into the jejunal and ileal loops of rats that were orally administered with NPWDQ, it was found that NPWDQ administration effectively diminished OVA permeation from both loops compared with that in rats that were not administered NPWDQ ${ }^{(6)}$. From these results, it was assumed that NPWDQ would enhance epithelial barrier function.

The epithelium maintains barrier function by the formation of complex protein-protein networks that mechanically link adjacent cells and seal the intercellular space. The protein

Abbreviations: ECM, extracellular matrix; EMC, enzyme-modified cheese; NPWDQ, Asn-Pro-Trp-Asp-Gln; OVA, ovalbumin; TER, transepithelial electrical resistance; TJ, tight junctions; ZO-1, zonula occludens-1.

* Corresponding author: Dr Soichi Tanabe, fax +81 82424 7916, email stanabe@ hiroshima-u.ac.jp 
networks that connect epithelial cells form three adhesive complexes: TJ, adherens and desmosomes. These complexes consist of transmembrane proteins that interact extracellularly with adjacent cells and intracellularly with adaptor proteins that link to the cytoskeleton ${ }^{(2)}$. For example, TJ consist of several proteins, and among them zonula occludens-1 (ZO-1) was the first to be identified. It binds to transmembrane proteins, such as claudins and occludin, and links them to cytoskeletal actin. TJ proteins are regulated by phosphorylation by kinases, phosphatases and other signalling molecules.

In the present study, we investigated the changes in the mRNA expression of genes related to $\mathrm{TJ}$ in Caco- 2 cells by the addition of NPWDQ using the microarray method. Western blot analyses of three major TJ proteins (occludin, claudin-1 and ZO-1) were also performed. We demonstrated that NPWDQ enforced TJ, at least partly, by increasing occludin expression.

\section{Materials and methods}

Caco-2 cells

Caco-2 cells were obtained from the American Type Culture Collection (Rockville, MD, USA). In the present study, cells were used between forty-five and fifty passages. The culture medium consisted of Dulbecco's modified Eagle's medium with $10 \%$ fetal bovine serum, $1 \%$ non-essential amino acids and antibiotics (100 units/ml penicillin, $100 \mu \mathrm{g} / \mathrm{ml}$ streptomycin and $50 \mu \mathrm{g} / \mathrm{ml}$ gentamycin). Dulbecco's modified Eagle's medium, non-essential amino acids, penicillin, streptomycin and gentamycin were all obtained from Invitrogen Corporation (Carlsbad, CA, USA). Fetal bovine serum was obtained from ICN Biomedicals, Inc. (Osaka, Japan).

Cells were cultured at $37^{\circ} \mathrm{C}$ under a humidified atmosphere of $5 \% \mathrm{CO}_{2}$. They were normally grown in $75 \mathrm{~cm}^{2}$ tissue culture flasks to approximately $80 \%$ confluence, and seeded into a twelve-well Transwell cell culture chamber $(0.4 \mu \mathrm{m}$ in pore size and $12 \mathrm{~mm}$ in diameter) (Corning Coster, Cambridge, MA, USA) at a density of $5 \times 10^{5}$ cells $/ \mathrm{cm}^{2}$. Each well was placed in a cluster plate with an outside medium (basolateral side, $1.5 \mathrm{ml}$ ) and an inside medium (apical side, $0.5 \mathrm{ml}$ ). The cell monolayers were fed fresh medium every $24 \mathrm{~h}$.

\section{Transepithelial electrical resistance measurement}

After $12-14 \mathrm{~d}$ of culture, transepithelial electrical resistance (TER) was measured using a Millicell-ERS instrument with $\mathrm{Ag} / \mathrm{AgCl}$ electrodes (Millipore, Bedford, MA, USA). Caco-2 cell monolayers were used whose TER values were $550-700 \Omega \mathrm{cm}^{2}$. NPWDQ was synthesised according to the solid-phase method using a peptide synthesiser, and was purified by reversed-phase HPLC by Takara Bio (Osaka, Japan).
To examine the effect of NPWDQ on TER, $0.5 \mathrm{ml}$ of the culture medium containing NPWDQ $\left(0,10^{-6}, 10^{-5}\right.$ and $\left.10^{-4} \mathrm{M}\right)$ was added to the apical side, and $1.5 \mathrm{ml}$ of fresh culture medium without NPWDQ was added to the basolateral side. Then, cells were incubated for $24 \mathrm{~h}$, after which, TER was measured.

\section{Microarray analysis}

After the above-mentioned experiments, RNA was extracted from Caco-2 cells, with or without incubation with NPWDQ $\left(10^{-6} \mathrm{M}\right)$, using a TRIzol reagent (Invitrogen) according to the manufacturer's instructions. RNA was extracted from six wells of the twelve-well Transwell cell culture chamber and pooled.

Complementary RNA was synthesised using an Illumina ${ }^{\circledR}$ RNA Amplification kit (Illumina, San Diego, CA, USA) according to the manufacturer's instructions. In brief, total RNA (150 ng) from Caco-2 cells was reverse transcribed with T7-oligo (dT) primer to synthesise first- and secondstrand complementary DNA, followed by clean-up. In vitro transcription was performed to synthesise biotin-labelled complementary RNA.

Labelled complementary RNA $(1.5 \mu \mathrm{g})$ was hybridised to a BeadChip (Sentrix Human WG-6; Illumina) at $58^{\circ} \mathrm{C}$ for $18 \mathrm{~h}$. Forty-eight thousand transcripts representing six wholegenome samples can be analysed on a single BeadChip. The hybridised BeadChip was washed and labelled with streptavidin-Cy3 (GE Healthcare Biosciences, Little Chalfont, Buckinghamshire, UK), and was scanned with an Illumina BeadStation 500GX-WG system (Illumina). Backgroundcorrected values for each probe on the BeadChip array were extracted using BeadStudio (Illumina). These results were expressed as an arbitrary unit, and analysed by GeneViewer (version 1.0; Moritex, Tokyo, Japan).

\section{Real-time RT-PCR}

The level of occludin mRNA ( $n$ 6) was measured with the real-time RT-PCR method using SYBR ${ }^{\circledR}$ green. The experiment was performed twice independently. Total RNA was treated with DNase (TURBO DNA-free ${ }^{\mathrm{TM}}$ kit; Applied Biosystems, Foster City, CA, USA). The RT reaction was conducted with MultiScribe ${ }^{\mathrm{TM}}$ reverse transcriptase using a high-capacity complementary DNA reverse transcription kit (Applied Biosystems) at $25^{\circ} \mathrm{C}$ for $10 \mathrm{~min}$ and at $37^{\circ} \mathrm{C}$ for $120 \mathrm{~min}$. The reaction was terminated by heating the reaction mixture at $85^{\circ} \mathrm{C}$ for $5 \mathrm{~s}$ followed by cooling at $4^{\circ} \mathrm{C}$.

Real-time RT-PCR was performed with a Real-Time PCR system (Applied Biosystems) using Power SYBR ${ }^{\circledR}$ Green PCR Master Mix (Applied Biosystems). Primers were designed using the Primer Express ${ }^{\circledR}$ program (Applied Biosystems). Their sequences are given in Table 1 . The reaction

Table 1. Sequences of primers used for real-time PCR

\begin{tabular}{lcll}
\hline & Amplicon length $(\mathrm{bp})$ & \multicolumn{1}{c}{ Oligonucleotide sequence } & GenBank accession number \\
\hline Occludin & 77 & $\begin{array}{l}\text { Forward: CCCATCTGACTATGTGGAAAGA } \\
\text { Reverse: AAAACCGCTTGTCATTCACTTTG }\end{array}$ & NM_002538 \\
3-Actin & 63 & $\begin{array}{l}\text { Forward: TCATGAAGTGTGACGTGGACATC } \\
\text { Reverse: TGCATCCTGTCGGCAATG }\end{array}$ & NM_001101 \\
\hline
\end{tabular}


was performed at $95^{\circ} \mathrm{C}$ for $10 \mathrm{~min}$, followed by forty cycles of $95^{\circ} \mathrm{C}$ for $15 \mathrm{~s}$ and $62^{\circ} \mathrm{C}$ for $1 \mathrm{~min}$. The dissociation stage was analysed at $95^{\circ} \mathrm{C}$ for $15 \mathrm{~s}$, followed by one cycle of $60^{\circ} \mathrm{C}$ for $15 \mathrm{~s}$ and $95^{\circ} \mathrm{C}$ for $15 \mathrm{~s}$. The fluorescence of the SYBR green dye was determined as a function of the PCR cycle number, giving the threshold cycle number at which amplification reached a significant threshold. Data were analysed by generating a standard curve from a dilution series and presented as fold change in gene expression, after normalisation of the $\beta$-actin gene.

\section{SDS-PAGE and Western blotting}

The expressions of occludin, claudin-1 and ZO-1 were examined by the ordinary method $(n 7)$. The experiment was performed twice independently. Briefly, Caco-2 cell monolayers were washed with ice-cold PBS thrice and lysed in the lysis buffer (25 mM Tris- $\mathrm{HCl}, 150 \mathrm{~mm} \mathrm{NaCl}, 1 \%$ Nonidet $\mathrm{P}-40,1 \%$ sodium deoxycholate and $1 \% \mathrm{SDS}$, at $\mathrm{pH} 7 \cdot 4)$. The cell suspensions were sonicated for $30 \mathrm{~min}$ using a sonicator (USK-3; As One Corporation, Osaka, Japan), and were centrifuged at $14000 \mathrm{~g}$ for $30 \mathrm{~min}$ at $4^{\circ} \mathrm{C}$ to yield a clear lysate. The proteins were separated using $12.5 \%$ gel for occludin and claudin-1 detection or $7.5 \%$ gel for ZO-1 detection, and were then transferred onto blotting membranes (Immobilon-P PVDF; Millipore). After overnight blocking with PBS/Tween 20 supplemented with $1 \%$ bovine serum albumin, the blots were incubated with primary (mouse anti-occludin, rabbit anti-claudin-1 or rabbit anti-ZO-1) and secondary antibodies (horseradish peroxidase-conjugated anti-mouse IgG or antirabbit $\mathrm{IgG}$, as appropriate) for $1 \mathrm{~h}$ each at room temperature. Anti-claudin-1, anti-occludin and anti-ZO-1 antibodies were all obtained from Zymed Laboratories (South San Francisco, CA, USA). Horseradish peroxidase-conjugated anti-mouse IgG was obtained from Sigma-Aldrich (St Louis, MO, USA). Anti-rabbit IgG was obtained from Kirkegaard and Perry Laboratories (Baltimore, MD, USA). To confirm equal loading, the blots were reprobed with rat anti- $\alpha$-tubulin antibody (Millipore). The proteins were visualised using chemiluminescence (enhanced chemiluminescence, GE Healthcare), and exposed to an X-ray film (Fujifilm, Kanagawa, Japan). Band densities were read using Scion Image Software (Scion, Frederick, MD, USA), and were normalised with $\alpha$-tubulin. SDS-PAGE and Western blotting were performed in duplicate.

\section{Statistical analyses}

All data are expressed as the means and standard deviations. Statistical analysis was performed using the Tukey-Kramer method.

\section{Results}

\section{Effect of Asn-Pro-Trp-Asp-Gln on transepithelial electrical resistance of Caco-2 monolayers}

The effect of the addition of NPWDQ on the integrity of Caco-2 monolayers was evaluated by the measurement of TER. TER value increased in response to the addition of increasing NPWDQ concentrations $\left(10^{-6}-10^{-4} \mathrm{M}\right)$, which suggests that this peptide enhanced epithelial barrier function (Fig. 1).

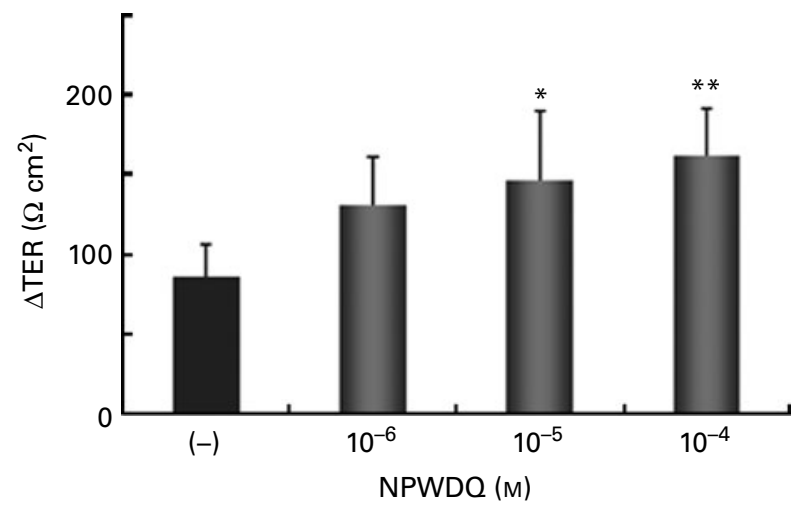

Fig. 1. Changes in transepithelial electrical resistance (TER) by the addition of Asn-Pro-Trp-Asp-GIn (NPWDQ) to Caco-2 cells. NPWDQ $\left(10^{-6}-10^{-4} \mathrm{M}\right)$ was added to the apical side, and cells were incubated for $24 \mathrm{~h}$, after which, TER was measured ( $n 6$ each). Mean values were significantly different for samples without the addition of NPWDQ (-): ${ }^{\star} P<0.05$, ${ }^{\star \star} P<0.01$.

\section{Microarray analysis of tight junction-related molecules}

DNA microarray analysis was performed to investigate the effect of the addition of NPWDQ in detail. NPWDQ $\left(10^{-6} \mathrm{M}\right)$ was added to the apical side of Caco- 2 cells, and the cells were incubated for $24 \mathrm{~h}$, after which, RNA was extracted. NPWDQ and the longer peptide Gly-Pro-Ile-ValLeu-Asn-Pro-Trp-Asp-Gln (GPIVLNPWDQ) (amino acids 102-111 of $\alpha s_{2}$-casein) significantly inhibited OVA permeation in Caco-2 cells at a concentration of $10^{-6} \mathrm{M}$ as shown previously ${ }^{(4)}$, so this concentration of NPWDQ was used in the present study.

Changes in 48000 types of gene expressions by NPWDQ were evaluated. To select genes with an abundant level of expression, those showing an expression of more than 1000 arbitrary fluorescence units, a total of 7108 genes were chosen and further analysed. Among these genes, only two genes, fos (GenBank accession number NM_005252) and egrl (NM_001964), were up-regulated by more than 2-fold, and three genes, mgc14376 (NM_032895), grb2 (NM_002086) and duspl (NM_004417), were up-regulated by 1.5-2-fold; the fold changes for fos, egrl, $\mathrm{mgcl} 4376$, grb2 and duspl were $2.93,2.92,1.54,1.53$ and 1.51, respectively. In addition, none of the genes was down-regulated by more than 1.5-fold (Table 2). Therefore, it can be suggested that generally NPWDQ did not drastically affect mRNA expression in Caco-2 cells.

Table 2. Summary of the changes in mRNA expressions in Caco-2 cells by the addition of Asn-Pro-Trp-Asp-GIn (NPWDQ) as evaluated by DNA microarray analysis

\begin{tabular}{lr}
\hline Fold change & Gene numbers \\
\hline$>1.5$ & 5 \\
$1.2-1.5$ & 122 \\
$0.8-1.2$ & 6850 \\
$0.67-0.8$ & 131 \\
$<0.67$ & 0 \\
\hline
\end{tabular}

NPWDQ $\left(10^{-6} \mathrm{M}\right)$ was added to the apical side, and cells were incubated for $24 \mathrm{~h}$. RNA was extracted from Caco-2 cells with or without incubation with NPWDQ (n 6 each), and pooled. Changes in 48000 types of gene expressions were evaluated.

In this table, the summary of 7108 genes that showed an expression of more than 1000 arbitrary fluorescence units is given. 
Table 3. Changes in the mRNA expressions of tight junction-related molecules by the addition of Asn-Pro-Trp-Asp-Gln (NPWDQ)

(Mean values and standard deviations)

\begin{tabular}{|c|c|c|c|c|c|c|c|c|}
\hline \multirow[b]{3}{*}{ Gene } & \multirow[b]{3}{*}{ GenBank accession number } & \multicolumn{6}{|c|}{ NPWDQ } & \multirow[b]{3}{*}{ Fold change } \\
\hline & & \multicolumn{3}{|c|}{$(-)$} & \multicolumn{3}{|c|}{$(+)$} & \\
\hline & & Mean & SD & $n$ & Mean & SD & $n$ & \\
\hline Occludin & NM_002538 & 164 & 34 & 36 & 245 & 43 & 31 & 1.49 \\
\hline Claudin-1 & NM_021101 & 23834 & 640 & 34 & 27039 & 790 & 32 & $1 \cdot 13$ \\
\hline Claudin-2 & NM_020384 & 1150 & 76 & 35 & 1041 & 43 & 44 & 0.91 \\
\hline Claudin-3 & NM_001306 & 2470 & 115 & 31 & 2557 & 105 & 41 & 1.04 \\
\hline Claudin-4 & NM_001305 & 624 & 57 & 30 & 588 & 35 & 45 & 0.94 \\
\hline Claudin-7 & NM_001307 & 11205 & 319 & 24 & 10881 & 329 & 42 & 0.97 \\
\hline Claudin-12 & NM_012129 & 3117 & 130 & 31 & 3225 & 121 & 48 & 1.03 \\
\hline Claudin-14 & NM_012130 & 3770 & 139 & 34 & 3303 & 141 & 37 & 0.88 \\
\hline Claudin-15 & NM_014343 & 3306 & 92 & 43 & 3487 & 133 & 37 & 1.05 \\
\hline Claudin-19 & NM_148960 & 531 & 35 & 51 & 587 & 52 & 39 & $1 \cdot 11$ \\
\hline Claudin-23 & NM_194284 & 4389 & 168 & 30 & 4492 & 220 & 23 & 1.02 \\
\hline ZO-1 & NM_003257 & 5398 & 133 & 63 & 5536 & 150 & 45 & 1.03 \\
\hline ZO-2 & NM_201629 & 2070 & 87 & 37 & 2223 & 79 & 41 & 1.07 \\
\hline ZO-3 & NM_014428 & 9363 & 286 & 39 & 8851 & 204 & 48 & 0.95 \\
\hline JAM-1 & NM_016946 & 8142 & 352 & 43 & 7834 & 264 & 36 & 0.96 \\
\hline
\end{tabular}

$n$, Number of beads analysed; ZO, zonula occludens; JAM-1, junctional adhesion molecule-1.

The expressions of claudin- $5,-6,-8,-9,-10,-11,-16,-17,-18,-20$ and -22 , and JAM-2 and -3 were $<100$ arbitrary fluorescence units.

Differences in gene expression of major TJ-related molecules, occludin, claudins and ZO, and junctional adhesion molecule families were analysed. As shown in Table 3, it was observed that occludin was up-regulated by 1.49 -fold. On the other hand, gene expressions of claudin-1, -2, -3, -4, -7, -12, -14, $-15,-19$ and -23 ; $\mathrm{ZO}$ family (ZO-1, -2 and -3 ) and junctional adhesion molecule-1 were not remarkably changed (fold changes, $0 \cdot 88-1 \cdot 13$ ). The genes of claudin-5, -6, -8, -9, -10, $-11,-16,-17,-18,-20$, and -22 , and junctional adhesion molecule-2 and -3 showed expressions at $<100$ arbitrary fluorescence units. From these data, it can be clearly suggested that NPWDQ up-regulated mRNA expression of occludin.

\section{Effect of Asn-Pro-Trp-Asp-Gln on occludin expression}

The effect of NPWDQ on the expression of the occludin gene was evaluated by real-time RT-PCR. As shown in Fig. 2, the addition of $10^{-6} \mathrm{M}$ NPWDQ increased occludin mRNA expression by $1 \cdot 3$-fold. To examine this phenomenon more clearly, increasing concentrations of NPWDQ $\left(10^{-5}\right.$ and $10^{-4} \mathrm{M}$ ) were added to the apical side of Caco- 2 cells, and mRNA expression of occludin was examined. As a result, NPWDQ $\left(10^{-5} \mathrm{M}\right)$ significantly $(P<0.01,1.7$-fold $)$ increased occludin mRNA expression. The addition of $10^{-4} \mathrm{M}$ NPWDQ also increased occludin mRNA expression, but the effect (1.4-fold) was less than that produced with the addition of $10^{-5} \mathrm{M}$ NPWDQ.

The effect of NPWDQ $\left(10^{-6} \mathrm{M}\right)$ on the expression of major TJ-related proteins, occludin, claudin-1 and ZO-1, was evaluated by Western blotting. NPWDQ significantly $(P<0 \cdot 05)$ increased the level of occludin by $1 \cdot 5$-fold (Fig. 3). Protein expressions of claudin-1 and ZO-1 were not changed by NPWDQ, which was consistent with the microarray data (Table 3). These data suggest that NPWDQ especially increased the protein level of occludin and enforced the TJ barrier.

\section{Discussion}

The intestine is constantly exposed to food components as well as to antigens, commensal microflora and pathogens. The epithelial TJ is therefore very important; several intestinal diseases have been reported to be associated with TJ dysfunction $^{(7)}$. The relationship between food components and the intestinal TJ barrier has not been completely elucidated, but information relating to glutamine ${ }^{(8)}, \mathrm{PUFA}^{(9)}, \mathrm{Zn}^{(10)}$ and polyphenols $^{(11)}$ is available. As for the modulation of occludin expression, Jiang et al. ${ }^{(9)}$ reported that occludin was up-regulated by $\gamma$-linolenic acid in the human vascular endothelial cell line ECV304. Following this, TER was increased, and paracellular permeability to large molecules was thereby reduced. To our knowledge, the present study is the first to show that occludin expression was up-regulated by a food-derived peptide.

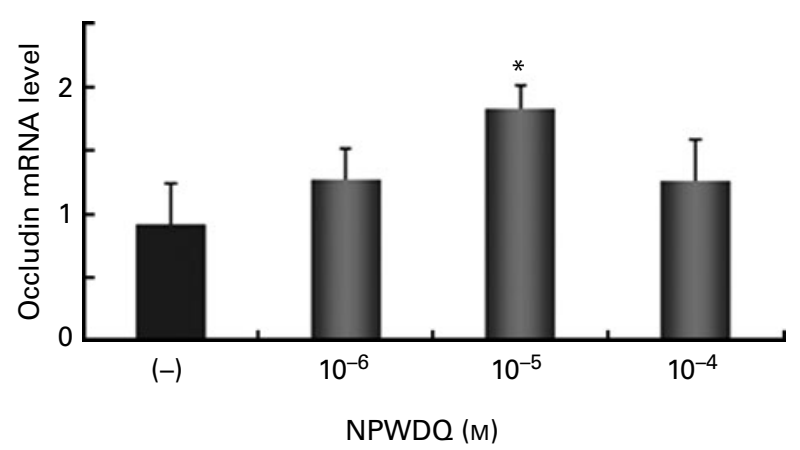

Fig. 2. Changes in the mRNA expression of occludin by the addition of AsnPro-Trp-Asp-GIn (NPWDQ) to Caco-2 cells. RNA was extracted from Caco-2 cells after the measurement of transepithelial electrical resistance (Fig. 1). The mRNA expression of occludin was evaluated by real-time RT-PCR ( $n 6$ each). * Mean values were significantly different for samples without the addition of NPWDQ $(-)(P<0.05)$. 
(A)
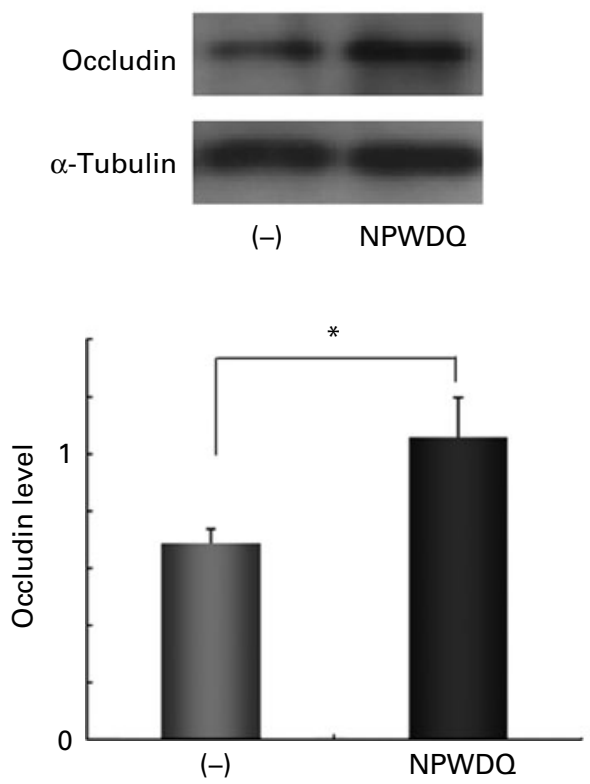

(B)

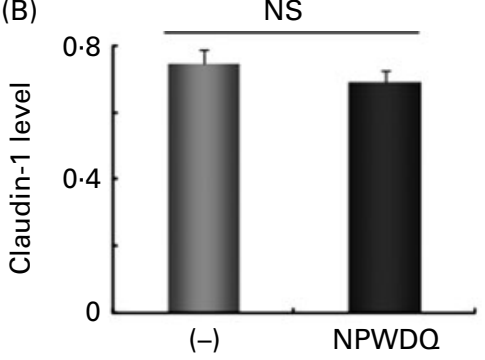

(C)

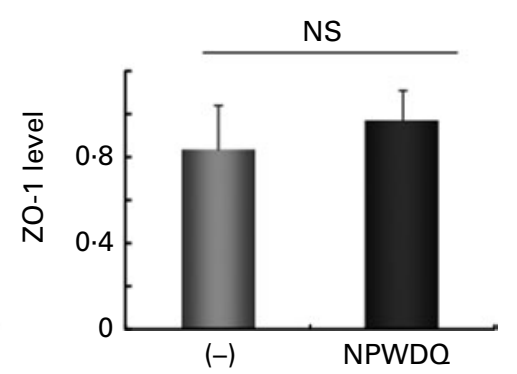

Fig. 3. Changes in the expressions of three tight junction proteins by the addition of Asn-Pro-Trp-Asp-GIn (NPWDQ) to Caco-2 cells. NPWDQ (10 $\left.{ }^{-6}{ }_{M}\right)$ was added to the apical side, and cells were incubated for $24 \mathrm{~h}$. Protein was extracted from Caco-2 cells with or without incubation with NPWDQ ( 7 each). Protein expressions were detected by Western blotting, and normalised with $\alpha$-tubulin for comparison. (a) Occludin (with a representative blotting pattern), (b) Claudin-1 and (c) Zonula occludens-1 (ZO-1). * Mean values were significantly different for samples without the addition of NPWDQ ( $)(P<0 \cdot 05)$.

Since we have reported previously that NPWDQ inhibited OVA permeation in Caco- 2 cells $24 \mathrm{~h}$ after the addition of the peptide ${ }^{(4)}$, we performed the DNA microarray analysis using RNA from Caco-2 cells that was incubated with the peptide for $24 \mathrm{~h}$. Indeed, the level of occludin mRNA expression did not change before $8 \mathrm{~h}$ incubation (data not shown). However, it would be interesting to examine whether the expression of other TJ molecules would change at an earlier stage ${ }^{(11)}$.

We demonstrated that NPWDQ up-regulated occludin expression in Caco-2 cells using the DNA microarray method followed by real-time RT-PCR and Western blot analyses. Occludin is a transmembrane protein of TJ, and it has an important role in the assembly and maintenance of $\mathrm{TJ}^{(11-14)}$. For example, the interaction between the $\mathrm{C}$-terminal region of occludin and ZO-1 is crucial for TJ assembly ${ }^{(13)}$. Therefore, up-regulation of occludin by NPWDQ might have a great impact on TJ in Caco-2 cells, although occludin was the only molecule whose expression level was significantly changed by NPWDQ. Further studies are necessary to examine the effect of NPWDQ on the localisation of other TJ proteins and on TJ structure. The effect of NPWDQ was the most prominent at a concentration of $10^{-5} \mathrm{M}$, and it reduced at $10^{-4} \mathrm{M}$ (Fig. 2). One possible explanation for this phenomenon is that in Caco- 2 cells a relatively high dose of the peptide might be recognised as a signal for ingested nutrients, and this might trigger a decrease in TER in order to absorb the peptide. Nevertheless, these data imply that a food-derived peptide can fine-tune the epithelial barrier by modulating the expression of $\mathrm{TJ}$ proteins such as occludin.

Is the effect of NPWDQ on occludin expression dependent on the peptide sequence? Or, are amino acids in the peptide sequence enough for the effect?
With respect to the activity of the constitutive amino acids (N, P, W, D and Q) towards the intestine, the protective effects of glutamine and tryptophan on the intestine have been characterised $^{(15-18)}$. Glutamine supplementation was demonstrated to improve intestinal barrier function in highly stressed patients $^{(15)}$ and in piglets with endotoxin-related changes in ileal permeability ${ }^{(16)}$. As for tryptophan, Kobayashi \& Watanabe $^{(17)}$ reported that its ethyl ester inhibited OVA permeation in the Caco-2 cell monolayer. Also, Kim et al. ${ }^{(18)}$ recently reported that tryptophan exhibited a therapeutic effect in piglets suffering from dextran sodium sulphate-induced colitis. Therefore, it is possible that NPWDQ would be hydrolysed into amino acids, and subsequently $\mathrm{P}$ and $\mathrm{W}$ would trigger the up-regulation of occludin. However, investigations are needed to ascertain if the existence of $\mathrm{P}$ and $\mathrm{W}$ residues is sufficient, or if the specific amino acid sequence is indispensable for the up-regulation of occludin.

Nevertheless, it is highly probable that NPWDQ would act as a peptide form because proline-containing peptides are, in general, resistant to degradation by digestive enzymes ${ }^{(19)}$. In this case, the next question is whether NPWDQ would be absorbed into Caco- 2 cells before its function inside cells or whether it is transported into the paracellular space to act from outside the cells. The underlying mechanisms of occludin up-regulation by NPWDQ are also unclear. It was reported that occludin was regulated by collagen IV, one of the components of the extracellular matrix (ECM), in TJ of Sertoli cells ${ }^{(20)}$. Savettieri et al. $^{(21)}$ also reported that ECM and neurons modulate the expression of occludin in rat brain endothelial cells. If NPWDQ were to be transported paracellularly and reacted with ECM, it would regulate occludin expression by reacting with molecules such as collagens in ECM. From our microarray data, it was found that NPWDQ 
did not drastically affect mRNA expressions of collagen IV$\alpha 3$ and $-\alpha 5$ (data not shown). Further studies are necessary to examine the effect of NPWDQ on ECM in intestinal cells.

As mentioned earlier, TJ dysfunction leads to intestinal disorders such as inflammatory bowel disease. For an in vitro model of inflammatory bowel disease, cytokine (e.g. TNF- $\alpha$ ) stimulated Caco- 2 cells are frequently used ${ }^{(22,23)}$. Upon stimulation, inflammatory cytokines, such as IL-8, are induced, and the TJ structure becomes loose. Several food components are under investigation for the treatment and/or prevention of inflammatory bowel disease using this cell model. For example, histidine was reported to inhibit TNF- $\alpha$-induced IL-8 secretion in Caco- 2 cells $^{(22)}$. Some types of lactobacilli also inhibited IL-8 production, and restored the protein expression of ZO-1 in TNF- $\alpha$-treated Caco- 2 cells ${ }^{(23)}$. It would be interesting to examine if NPWDQ up-regulates occludin expression in such inflamed intestinal cells.

For practical intake of NPWDQ, food materials comprising this type of peptide are more acceptable to the industry than synthetic peptides. In this context, EMC seems to be favourable. We have found NPWDQ peptide from EMC that has an inhibitory activity towards allergen absorption in the intestine ${ }^{(4,6)}$. EMC is generally produced by the hydrolysis of cheese with commercial proteases, and it is currently used in the food industry. We hope that EMC will be used for the treatment of food allergy and intestinal disorders or for an intestinal barrier-promoting purpose in healthy people.

In conclusion, a casein-derived peptide NPWDQ, which has inhibitory effects against allergen permeation in the intestine, up-regulates occludin expression and enforces the TJ barrier. These data imply that a food-derived peptide can fine-tune the epithelial barrier.

\section{Acknowledgements}

The present study was partially supported by the Food Science Institute Foundation (Ryousyoku-kenkyukai, Kanagawa, Japan). We thank Dr Takuya Suzuki (Hiroshima University) for his valuable discussions. We also thank Dr Noriyuki Yanaka (Hiroshima University) for his technical support. The authors' responsibilities are as follows: H. Y. conducted all the experiments. S. T. designed the study and wrote the manuscript. Both the authors approved the final manuscript. The authors declare that there is no conflict of interest.

\section{References}

1. Kaminogawa S, Hachimura S, Nakajima-Adachi H, et al. (1999) Food allergens and mucosal immune systems with special reference to recognition of food allergens by gut-associated lymphoid tissue. Allergol Int 48, 15-23.

2. Groschwitz KR \& Hogan SP (2009) Intestinal barrier function: molecular regulation and disease pathogenesis. J Allergy Clin Immunol 124, 3-20.

3. Ventura MT, Polimeno L, Amoruso AC, et al. (2006) Intestinal permeability in patients with adverse reactions to food. Dig Liver Dis 38, 732-736.

4. Tanabe S, Isobe N, Miyauchi E, et al. (2006) Identification of a peptide in enzymatic hydrolyzate of cheese that inhibits ovalbumin permeation in Caco-2 cells. J Agric Food Chem 54, 6904-6908.
5. Kobayashi S, Tanabe S, Sugiyama M, et al. (2008) Transepithelial transport of hesperetin and hesperidin in intestinal Caco-2 cell monolayers. Biochim Biophys Acta 1778, 33-41.

6. Isobe N, Suzuki M, Oda M, et al. (2008) Enzyme-modified cheese exerts inhibitory effects on allergen permeation in rats suffering from indomethacin-induced intestinal inflammation. Biosci Biotechnol Biochem 72, 1740-1745.

7. Amasheh M, Andres S, Amasheh, et al. (2009) Barrier effects of nutritional factors. Ann N Y Acad Sci 1165, 267-273.

8. Li N, Lewis P, Samuelson D, et al. (2004) Glutamine regulates Caco-2 cell tight junction proteins. Am J Physiol Gastrointest Liver Physiol 287, G726-G733.

9. Jiang WG, Bryce RP, Horrobin DF, et al. (1998) Regulation of tight junction permeability and occludin expression by polyunsaturated fatty acids. Biochem Biophys Res Commun 244, 414-420.

10. Finamore A, Massimi M, Conti Devirgiliis L, et al. (2008) Zinc deficiency induces membrane barrier damage and increases neutrophil transmigration in Caco-2 cells. $J$ Nutr 138, 1664-1670.

11. Suzuki T \& Hara H (2009) Quercetin enhances intestinal barrier function through the assembly of zonula occludens-2, occludin, and claudin- 1 and the expression of claudin- 4 in Caco- 2 cells. J Nutr 139, 965-974.

12. Rao R (2009) Occludin phosphorylation in regulation of epithelial tight junctions. Ann N Y Acad Sci 1165, 62-68.

13. Suzuki T, Elias BC, Seth A, et al. (2009) PKC eta regulates occludin phosphorylation and epithelial tight junction integrity. Proc Natl Acad Sci U S A 106, 61-66.

14. Qin H, Zhan Z, Hang X, et al. (2009) L. plantarum prevents enteroinvasive Escherichia coli-induced tight junction proteins changes in intestinal epithelial cells. BMC Microbiol 9, 63.

15. Neu J, DeMarco V \& Li N (2002) Glutamine: clinical applications and mechanisms of action. Curr Opin Clin Nutr Metab Care 5, 69-75.

16. Dugan ME \& McBurney MI (1995) Luminal glutamine perfusion alters endotoxin-related changes in ileal permeability of the piglet. $J$ Parenter Enteral Nutr 19, 83-87.

17. Kobayashi S \& Watanabe J (2003) Inhibitory activities of aromatic amino acid esters and peptides against ovalbumin permeation through Caco-2 cell monolayers. Biosci Biotechnol Biochem 67, 2498-2500.

18. Kim CJ, Kovacs-Nolan JA, Yang C, et al. (2009) L-Tryptophan exhibits therapeutic function in a porcine model of dextran sodium sulfate (DSS)-induced colitis. J Nutr Biochem (epublication ahead of print version 8 May 2009).

19. Vermeirssen V, Van Camp J \& Verstraete W (2004) Bioavailability of angiotensin I converting enzyme inhibitory peptides. Br J Nutr 92, 357-366.

20. Siu MK, Lee WM \& Cheng CY (2003) The interplay of collagen IV, tumor necrosis factor-alpha, gelatinase B (matrix metalloprotease-9), and tissue inhibitor of metalloproteases-1 in the basal lamina regulates Sertoli cell-tight junction dynamics in the rat testis. Endocrinology 144, 371-387.

21. Savettieri G, Di Liegro I, Catania C, et al. (2000) Neurons and ECM regulate occludin localization in brain endothelial cells. Neuroreport 11, 1081-1084.

22. Son DO, Satsu H \& Shimizu M (2005) Histidine inhibits oxidative stress- and TNF-alpha-induced interleukin-8 secretion in intestinal epithelial cells. FEBS Lett 579, 4671-4677.

23. Miyauchi E, Morita H, Okuda J, et al. (2008) Cell wall fraction of Enterococcus hirae ameliorates TNF-alpha-induced barrier impairment in the human epithelial tight junction. Lett Appl Microbiol 46, 469-476. 